\title{
STAR FORMATION IN COLLISIONS BETWEEN TWO GAS- RICH DISK GALAXIES
}

\author{
CURTIS STRUCK \\ Iowa State University \\ Dept. of Physics and Astronomy \\ Ames, IA 50011 USA
}

We present the results of a modest grid of three-dimensional (SPH) simulations of nearly head-on collisions between two model galaxies, each consisting of a rigid halo and a gas-rich disk component. The companion to primary mass ratio used is typically $0.2-0.35$. Simple models of radiative cooling and heating from young star activity are also included in the simulations. Star formation is assumed to occur when the local gas density exceeds a threshold value. In these collisions the primary gas disk remains largely intact, though a gas bridge is splashed out between the two galaxies. Star formation occurs in asymmetric ring-like waves in the primary. The bridge is predicted to have essentially no ongoing star formation. These results agree with observations of the Cartwheel and VII Zw 466 ring galaxies.

The companion gas disk is usually disrupted by the impact, but reforms via accretion from the bridge. Star formation is often delayed in the companion until the gas disk has reformed. Interestingly, star formation can also be reduced or delayed in the central regions of the primary, apparently as a result of accretion heating. For example, in models with a diskless companion star formation is strongest in the first ring wave, while in some models with a companion gas disk the third ring, which forms at the end of the accretion phase, is strongest. These results suggest the general possibility that heating due to high rates of accretion is able to delay starbursts in collisional systems. For further details see Struck (1997, and Struck et al. 1996, for applications to the inner ring of the Cartwheel).

\section{References}

Struck, C. 1997, $A p J S, \mathbf{1 1 3}$, in press

Struck, C., Appleton, P.N., Borne, K.D., \& Lucas, R.A. 1996, AJ, 112, 1868 